Published in final edited form as:

Am J Kidney Dis. 2013 July ; 62(1): 165-178. doi:10.1053/j.ajkd.2012.12.022.

\title{
Biomarkers in Nephrology
}

\author{
Gearoid M. McMahon, MB, BCh and Sushrut S. Waikar, MD, MPH \\ Renal Division, Department of Medicine, Brigham and Women's Hospital, Harvard Medical \\ School, Boston, MA
}

The clinical assessment and management of patients with known or suspected kidney disease has been aided for decades by biomarkers, a term defined by a National Institutes of Health (NIH) working group as "A characteristic that is objectively measured and evaluated as an indicator of normal biological processes, pathogenic processes, or pharmacological responses to a therapeutic intervention." The characteristics of an ideal biomarker are given in Box 1. Historically, the first biomarker of kidney disease was the finding on physical examination of interstitial edema or ascites, a condition termed dropsy, that was not specific to what eventually became recognized as kidney failure but that rather encompassed a number of clinical conditions including congestive heart failure and cirrhosis. More objective biomarkers in the early days of nephrology included the examination of the urine sediment, followed by measurement of the blood urea nitrogen concentration and the serum creatinine concentration.

Recently, there has been an explosive growth in the search for more sensitive, specific, and prognostically accurate biomarkers to assist in the care of patients with or at risk of kidney disease. In this review we aim to discuss both conventional and novel biomarkers of kidney disease in the settings of acute kidney injury (AKI), chronic kidney disease (CKD), nephrotoxin exposure, and glomerulonephritis. An anatomical localization of these biomarkers along the nephron is shown in figure 1 .

\section{Additional Readings}

\section{References}

1. Biomarkers Definitions Working Group. Biomarkers and surrogate endpoints: Preferred definitions and conceptual framework. Clin Pharmacol Ther. 2001; 69(3):89-95. [PubMed: 11240971]

\section{CONVENTIONAL BIOMARKERS OF KIDNEY FUNCTION AND DISEASE}

Modern definitions of CKD and AKI emphasize a stage of decreased glomerular filtration rate (GFR), and a stage of kidney damage, defined as structural or functional abnormalities before a decline in GFR. Corresponding biomarkers include filtration markers, such as

(C) 2013 The National Kidney Foundation, Inc. Published by Elsevier Inc. All rights reserved.

Correspondence to: Sushrut S. Waikar, MD, MPH, Brigham and Women's Hospital, MRB-4, 75 Francis Street, Boston, MA 02115, 617-732-8474 (office), 617-732-6392 (fax), swaikar@partners.org.

Financial Disclosure: Dr. Waikar has served as a consultant to CVS Caremark, BioTrends Research Group, and Takeda; provided expert testimony for GE Heathcare, Northstar Rx, and Salix; and received grants from the National Institute of Diabetes and Digestive Kidney Diseases, Otsuka, Merck, Genzyme, and Satellite Healthcare. Dr. McMahon declares that he has no relevant financial interests.

Publisher's Disclaimer: This is a PDF file of an unedited manuscript that has been accepted for publication. As a service to our customers we are providing this early version of the manuscript. The manuscript will undergo copyediting, typesetting, and review of the resulting proof before it is published in its final citable form. Please note that during the production process errors may be discovered which could affect the content, and all legal disclaimers that apply to the journal pertain. 
creatinine and cystatin $\mathrm{C}$, and markers of kidney damage, such as urine sediment abnormalities and albuminuria (Table 1).

\section{Creatinine}

The use of creatinine as a marker of GFR dates back to the 1920s. Creatinine is a $113 \mathrm{Da}$ protein that is the product of non-enzymatic breakdown of creatine in muscle. It is not protein bound, not metabolized in the kidney, and is freely filtered in the glomerulus, making it an excellent marker of glomerular filtration. However, non-GFR determinants of creatinine concentration limit its utility in AKI and CKD. Proximal tubular secretion of creatinine accounts for 10-20\% of its excretion leading to an overestimation of the true GFR, particularly in patients with CKD. Gut bacteria also degrade creatinine and contribute to its clearance, the relative magnitude of which becomes more important as kidney function declines. Creatinine can be reabsorbed after glomerular filtration in patients with very low urine and tubular flow rates. Certain medications such as cimetidine and trimethoprim can increase the serum creatinine concentration by inhibiting tubular secretion. Creatinine is produced at a relatively constant rate, which is in turn proportional to muscle mass. Between-person variability in creatinine generation rate-related to age, sex, muscle mass, race, and perhaps other factors-limits the use of creatinine in the estimation of GFR. To account for this variability, a number of creatinine-based equations have been developed to estimate GFR, including the Cockroft-Gault, Modification of Diet in Renal Disease (MDRD) Study, and CKD-EPI (Chronic Kidney Disease Epidemiology Collaboration) equations for adults, and the Schwartz equation for children. Despite improving the estimation of true GFR, all the equations have shortcomings. For example, at lower creatinine concentrations the MDRD equation generally underestimates the GFR, whereas the Cockroft-Gault and Schwartz equations may overestimate the GFR.

\section{Additional Readings}

\section{References}

2. Carrie BJ, Golbetz HV, Michaels AS, Myers BD. Creatinine: An inadequate filtration marker in glomerular diseases. Am J Med. 1980; 69(2):177-182. [PubMed: 6157324]

3. Hankins DA, Babb AL, Uvelli DA, Scribner BH. Creatinine degradation I: The kinetics of creatinine removal in patients with chronic kidney disease. Int J Artif Organs. 1981; 4(1):35-39. [PubMed: 7216531]

4. Perrone RD, Madias NE, Levey AS. Serum creatinine as an index of renal function: New insights into old concepts. Clin Chem. 1992; 38(10):1933-1953. [PubMed: 1394976]

5. Rehberg PB. Studies on kidney function: The rate of filtration and reabsorption in the human kidney. Biochem J. 1926; 20(3):447-460. [PubMed: 16743679]

\section{Cystatin C}

Cystatin CC is a 13-kDa cysteine protease inhibitor that is a marker of GFR. Cystatin C is expressed by all nucleated cells and has multiple biologic functions including controlling extracellular proteolysis and modulation of the immune system. Its utility in estimating kidney function derives from the fact that after being freely filtered in the glomerulus, it is then absorbed in the kidney tubules where it is fully degraded locally.

There is no active tubular secretion or significant extrarenal elimination making it an excellent marker of GFR. Cystatin C may also be superior to creatinine as a better predictor of cardiovascular mortality while providing a more accurate estimation of GFR.

The serum cystatin $\mathrm{C}$ concentration is independent of muscle mass, nutritional status, and sex, although it may be altered in patients with derangements in thyroid function, certain 
cancers, and with glucocorticoid therapy. Although the production of cystatin $\mathrm{C}$ has been well characterized in healthy individuals, less is known about its production in disease states; for example, cystatin $\mathrm{C}$ levels are higher at baseline in patients with acute leukemia. There are a number of different methods for measuring cystatin $\mathrm{C}$ and there is significant interassay variation, with some assays performing better for the diagnosis of AKI and CKD.

A major advantage of cystatin $\mathrm{C}$ over creatinine is that it is not as influenced by changes in muscle mass; therefore estimating equations for eGFR are more accurate across a range of body types including infants and the elderly. In patients at extremes of body mass, creatinine-based equations perform poorly while cystatin $\mathrm{C}$ is more accurate. However, the increase in accuracy is moderate at best, and it is not certain that it is clinically relevant. Some have proposed combining creatinine and cystatin $\mathrm{C}$ equations for more precise estimations of GFR. In this setting, agreement between the two estimates can be taken as a more accurate estimation of GFR. However, if there is disagreement, the clinical picture should be considered to determine the source of error (body mass, corticosteroids etc.). A recent study has suggested that the combination of creatinine and cystatin $\mathrm{C}$ for the estimation of GFR is superior to creatinine-based equations and may be useful as a confirmatory test for CKD.

Cystatin $\mathrm{C}$ is distributed in the extracellular space while creatinine is distributed in the total body water. As a result, it has a volume of distribution approximately $1 / 3$ that of creatinine, meaning that it reaches a steady state concentration 3 times faster: the half-life is 1.5 hours vs. 4 hours. As a result, following kidney injury, the cystatin $\mathrm{C}$ concentration increases earlier than creatinine. This has been borne out in studies of patients with AKI including contrast-induced nephropathy where the rise in cystatin $\mathrm{C}$ precedes that of creatinine by between 5 and 24 hours. In a large study of patients undergoing cardiac surgery, however, cystatin $\mathrm{C}$ did not exhibit favorable kinetic characteristics compared to serum creatinine in the diagnosis of AKI.

Urinary cystatin $\mathrm{C}$ is also a potential marker of acute tubular injury. Because cystatin $\mathrm{C}$ is usually reabsorbed in the proximal tubule, the finding of cystatin $\mathrm{C}$ in the urine suggests some form of proximal tubular injury. However, in patients with albuminuria, there is competitive inhibition of cystatin $\mathrm{C}$ uptake (both are transported by megalin), and as a result, significant quantities can appear in the urine even in the absence of tubular injury. This effect has been demonstrated in rats, and similar effects have been noted in patients with diabetes and in children with the nephrotic syndrome. It should be noted that cystatin $\mathrm{C}$ is not the only urinary biomarker that is affected by increasing levels of albuminuria: all of the low molecular weight proteins under investigation as potential urinary biomarkers could potentially be affected by this process including neutrophil gelatinase-associated lipocalin (NGAL), liver-type fatty-acid binding protein (L-FABP), and $\beta 2$ microglobulin.

\section{Additional Readings}

\section{References}

6. Briguori C, Visconti G, Rivera NV, et al. Cystatin C and contrast-induced acute kidney injury. Circulation. 2010; 121(19):2117-2122. [PubMed: 20439784]

7. Demirtas S, Akan O, Can M, Elmali E, Akan H. Cystatin C can be affected by nonrenal factors: A preliminary study on leukemia. Clin Biochem. 2006; 39(2):115-118. [PubMed: 16337174]

8. Edelstein, CL. Biomarkers in kidney disease. 1. Amsterdam ; Boston: Academic Press/Elsevier; 2011.

9. Grubb A. Diagnostic value of analysis of cystatin $\mathrm{C}$ and protein $\mathrm{HC}$ in biological fluids. Clin Nephrol. 1992; 38(Suppl 1):S20-7. [PubMed: 1284235] 
10. Herget-Rosenthal S, Marggraf G, Husing J, et al. Early detection of acute renal failure by serum cystatin C. Kidney Int. 2004; 66(3):1115-1122. [PubMed: 15327406]

11. Inker LA, Schmid CH, Tighiouart $\mathrm{H}$, et al. Estimating glomerular filtration rate from serum creatinine and cystatin C. N Engl J Med. 2012; 367(1):20-29. [PubMed: 22762315]

12. Manzano-Fernandez S, Januzzi JL Jr, Boronat-Garcia M, et al. Beta-trace protein and cystatin C as predictors of long-term outcomes in patients with acute heart failure. J Am Coll Cardiol. 2011; 57(7):849-858. [PubMed: 21310322]

13. Nejat M, Hill JV, Pickering JW, Edelstein CL, Devarajan P, Endre ZH. Albuminuria increases cystatin C excretion: Implications for urinary biomarkers. Nephrol Dial Transplant. 2011

14. Nejat M, Pickering JW, Walker RJ, Endre ZH. Rapid detection of acute kidney injury by plasma cystatin $C$ in the intensive care unit. Nephrol Dial Transplant. 2010; 25(10):3283-3289. [PubMed: 20350927]

15. Priem F, Althaus H, Jung K, Sinha P. Beta-trace protein is not better than cystatin C as an indicator of reduced glomerular filtration rate. Clin Chem. 2001; 47(12):2181. [PubMed: 11719491]

16. Shlipak MG, Sarnak MJ, Katz R, et al. Cystatin C and the risk of death and cardiovascular events among elderly persons. N Engl J Med. 2005; 352(20):2049-2060. [PubMed: 15901858]

17. Spahillari A, Parikh CR, Sint K, et al. Serum cystatin C- versus creatinine-based definitions of acute kidney injury following cardiac surgery: A prospective cohort study. Am J Kidney Dis. 2012

\section{Urine Sediment Analysis}

The examination of the urine sediment is a time-honored test relied upon by generations of nephrologists to aid in the diagnosis of kidney diseases. The actual diagnostic performance characteristics of the urine sediment examination and its inter- and intra-observer variability have not been well characterized. The presence of specific findings in the urine sediment can aid in the diagnosis of acute and chronic kidney disease prior to more invasive testing. Examination of the urine sediment can help discriminate proliferative glomerular disease from non-proliferative diseases. Hematuria and red cell casts are a sensitive early sign of relapse in patients with lupus nephritis. White blood cell casts may be seen in tubulointerstitial nephritis, but may also been seen in glomerulonephritis and pyelonephritis. Muddy brown casts, granular casts, and kidney tubular epithelial cells and casts can indicate acute tubular injury, but can be seen in a variety of other acute and chronic kidney diseases.

In the setting of AKI, recent carefully performed studies have suggested that a semiquantitative scoring system based on the number of kidney tubular epithelial cells and granular casts may provide useful information on the differential diagnosis and prognosis of AKI (Table 2). The combination of this score with novel biomarkers including kidney injury molecule-1 (KIM-1), NGAL, and interleukin-18 (IL-18) has been shown to be more sensitive than creatinine or urine electrolytes alone in predicting the need for dialysis in patients with AKI.

However, the diagnostic utility of urine sediment analysis results in clinical practice are uncertain due to a lack of standardization in the reporting of results and the potential for significant inter- and intra-observer variability. Most of the studies of the performance of the urine sediment involve clinicians with many years of experience and an interest in examining urine sediments. This may contrast with the experience of the general population of practicing nephrologists, internists and trainees. There is a need for a single standard for reporting urine sediment analysis results that correlates with the results of kidney biopsies and clinical outcomes. Future studies are needed to quantify the diagnostic characteristics of the urine sediment examination in a variety of kidney diseases and to assess the inter- and intra-observer reliability. 


\section{Additional Readings}

References

18. Fogazzi GB, Ponticelli C, Ritz E. The urine sediment: an integrated view. Elsevier. 2010

19. Hall IE, Coca SG, Perazella MA, et al. Risk of poor outcomes with novel and traditional biomarkers at clinical AKI diagnosis. Clin J Am Soc Nephrol. 2011

20. Perazella MA, Coca SG. Traditional urinary biomarkers in the assessment of hospital-acquired AKI. Clin J Am Soc Nephrol. 2011

21. Perazella MA, Coca SG, Hall IE, Iyanam U, Koraishy M, Parikh CR. Urine microscopy is associated with severity and worsening of acute kidney injury in hospitalized patients. Clin J Am Soc Nephrol. 2010; 5(3):402-408. [PubMed: 20089493]

\section{Albuminuria}

Proteinuria has long been recognized as a consequence of kidney damage. The measurement of urinary protein and urinary albumin is a central component of screening for and monitoring of CKD. The glomerulus acts as a barrier to filtration due to pore size and charge selectivity, while the majority of filtered albumin is reabsorbed in the proximal tubule. Thus, the presence of albuminuria usually indicates damage to the filtration barrier but may also be the result of proximal tubular dysfunction.

Multiple studies have demonstrated that albuminuria is an independent risk factor for mortality in the general population. Albuminuria is also an independent risk factor for the progression of diabetic and non-diabetic CKD. As a result, it has been proposed that stage III CKD be further stratified by the presence or absence of albuminuria. The reduction of albuminuria is a commonly accepted goal of treatment in patients with CKD, although it is not as yet an acceptable surrogate endpoint in clinical trials. However, the FDA recently qualified its use as biomarker of proximal tubular injury from nephrotoxin exposure in animal studies.

\section{Additional Readings}

\section{References}

22. Matsushita K, van der Velde M, et al. Chronic Kidney Disease Prognosis Consortium. Association of estimated glomerular filtration rate and albuminuria with all-cause and cardiovascular mortality in general population cohorts: A collaborative meta-analysis. Lancet. 2010; 375(9731):2073-2081. [PubMed: 20483451]

23. Levey AS, de Jong PE, Coresh J, et al. The definition, classification, and prognosis of chronic kidney disease: A KDIGO controversies conference report. Kidney Int. 2011; 80(1):17-28. [PubMed: 21150873]

\section{NOVEL BIOMARKERS}

The primary rationale for the development of novel biomarkers of kidney diseases is that early and more accurate diagnosis and/or prognosis may allow for interventions that prevent progression. Because current biomarkers of kidney disease become elevated relatively late in the injury process, they do not allow for early interventions that could be successful at preventing propagation of the injury. Early identification could allow for injury-specific interventions that are currently not possible. This could be particularly useful in AKI when a defined insult - e.g., following contrast administration or cardiac surgery - could allow for more appropriate risk stratification and treatment (Table 3). Thus, novel biomarkers have a number of potential roles in nephrology:

1. To more accurately diagnose AKI, early in the course of the disease

2. To correlate better with GFR in patients with CKD 
3. To determine the anatomic location of injury in patients with AKI (i.e. glomerular, tubular, interstitial or vascular)

4. To identify the cause of AKI and CKD

5. To monitor the effectiveness of interventions

6. To provide information regarding the prognosis of AKI and CKD.

One significant issue surrounding the development of biomarkers in nephrology is the frequent absence of a reliable gold standard for the diagnosis of kidney disease. The limitations of creatinine as a biomarker were discussed above, but in brief, it is insensitive, not very specific, and tends to rise relatively late in the course of kidney injury when the injury may not be reversible. Currently, the commonly used definitions of AKI (RIFLE, AKIN [Acute Kidney Injury Network], and KDIGO [Kidney Disease: Improving Global Outcomes]) incorporate creatinine concentration into the diagnostic framework, while equations for estimating GFR in patients with CKD (MDRD Study equation and CKD-EPI equation) also generally require creatinine.

It is notable that recent studies have demonstrated the usefulness of novel biomarkers in mild AKI. Urinary levels of certain biomarkers have been found to be elevated in patients with pre-renal AKI (Kim-1, cystatin C and IL-18) while others (NGAL, $\gamma$-glutamyl transpeptidase [GGT]) were not. A recent meta-analysis found that an elevated urinary or plasma NGAL predicted the future need for renal replacement therapy and in-hospital mortality in critically ill patients in the intensive care unit, even in the absence of a clinically significant rise in creatinine. This suggests that there is a spectrum of subclinical but significant AKI that is not being detected by the traditional gold standard methods.

In animal studies, increasing levels of kidney injury biomarkers correlate with the gold standard of increasing structural damage on kidney biopsy in models of AKI. However, there have been no large studies in humans correlating the structural appearance with biomarker levels. As a result, we continue to use creatinine as the gold standard in biomarker trials. However, biomarker levels may relate to the severity of kidney injury and be associated with the occurrence of clinical outcomes including need for dialysis and death. Recently, the FDA qualified a set of urinary biomarkers of nephrotoxicity for regulatory use in certain pre-clinical settings (i.e., animal studies). These included urinary KIM-1, albumin, total protein, albumin, $\beta 2$-microglobulin, cystatin $\mathrm{C}$, clusterin, trefoil factor-3, and renal papillary antigen-1. The recent FDA qualification of novel injury biomarkers demonstrates a move away from creatinine as the gold standard for studies in nephrology towards a more nuanced approach.

\section{Additional Readings}

References

24. Dieterle F, Sistare F, Goodsaid F, et al. Renal biomarker qualification submission: A dialog between the FDA-EMEA and predictive safety testing consortium. Nat Biotechnol. 2010; 28(5): 455-462. [PubMed: 20458315]

25. Edelstein, CL. Biomarkers in kidney disease. 1. Amsterdam ; Boston: Academic Press/Elsevier; 2011.

26. Endre ZH, Pickering JW, Walker RJ. Clearance and beyond: The complementary roles of GFR measurement and injury biomarkers in acute kidney injury (AKI). Am J Physiol Renal Physiol. 2011; 301(4):F697-707. [PubMed: 21753074] Haase M, Devarajan P, Haase-Fielitz A, et al. The outcome of neutrophil gelatinase-associated lipocalin-positive subclinical acute kidney injury: A multicenter pooled analysis of prospective studies. J Am Coll Cardiol. 2011; 57(17):1752-1761. [PubMed: 21511111] 
27. Nejat M, Pickering JW, Devarajan P, et al. Some biomarkers of acute kidney injury are increased in pre-renal acute injury. Kidney Int. 2012

28. Ozer JS, Dieterle F, Troth S, et al. A panel of urinary biomarkers to monitor reversibility of renal injury and a serum marker with improved potential to assess renal function. Nat Biotechnol. 2010; 28(5):486-494. [PubMed: 20458319]

\section{Plasma/serum vs. Urinary Biomarkers}

The urine and the plasma/serum are potential sources of biomarkers in nephrology. Both are easily collected and are minimally invasive. Urine has a number of advantages over serum for the evaluation of potential biomarkers. Urine is a proximal fluid, meaning that it is in close contact to the site of injury (the kidney), and as such, is a site where biomarkers can accumulate after being shed by damaged kidney tissue. In patients with AKI, urinary biomarkers increase relatively early in the course of the disease. In contrast, for serum markers of GFR, such as creatinine and cystatin C, there is a significant lag between the time of injury and the time that their concentrations will exceed the threshold required to make a diagnosis of AKI. Urinary biomarkers can be separated into those that are present in the kidney and released in the event of damage (e.g. proximal tubular enzymes), and those induced by tubular injury (e.g., KIM-1, NGAL, and IL-18). Preformed biomarkers appear in the urine shortly after injury while there is a time lag for the appearance of the induced biomarkers.

Urine biomarkers have a number of important potential limitations. It is important that the urine is handled correctly. Urinary biomarkers may not be stable for long periods of time and urine should be promptly frozen if it is not immediately analyzed. There is a potential for error if the urine is not collected appropriately. Mid-stream urine samples are more informative in women, presumably because initial voids are more prone to bacterial contamination. In contrast, for males, prostate cancer markers are more readily detectable in first-void urine samples. Baseline kidney function is an important modifying influence. As detailed below, a major limitation of urinary biomarkers is the fact that their concentration changes with hydration status and multiple methods of dealing with this problem have been suggested, including normalizing to the urinary creatinine concentration.

Serum or plasma is also a good potential source of biomarkers. It is obtainable in anuric patients and less prone to bacterial contamination and breakdown of its constituents. However, changes in serum biomarker concentrations are not necessarily related to reduced kidney function alone and can be the product of a systemic response. The large number of proteins present in the serum makes the discovery of specific kidney markers more complicated.

\section{Additional Readings}

\section{References}

29. Edelstein, CL. Biomarkers in kidney disease. 1. Amsterdam ; Boston: Academic Press/Elsevier; 2011.

30. Hewitt SM, Dear J, Star RA. Discovery of protein biomarkers for renal diseases. J Am Soc Nephrol. 2004; 15(7):1677-1689. [PubMed: 15213255]

31. Wu J, Chen YD, Gu W. Urinary proteomics as a novel tool for biomarker discovery in kidney diseases. J Zhejiang Univ Sci B. 2010; 11(4):227-237. [PubMed: 20349519]

\section{Normalization of Urinary Biomarkers}

Because the kidney has the ability to reabsorb water over a wide range, the concentration of a biomarker in the urine depends not only on its rate of production but also on the urine flow rate. Because of this, absolute values of urine biomarkers will change significantly 
depending on the urine flow rate, thus making them difficult to interpret. Ideally, urinary biomarkers should be normalized by expressing them as a ratio to a substance that is excreted at a constant rate by the kidneys. In practice, urinary biomarkers are commonly expressed as a ratio to the urinary creatinine concentration. This assumes that creatinine production is relatively constant across individuals and relative to the urinary biomarker concentrations in healthy and diseased states. However, the urine creatinine concentration is affected by a number of factors including the creatinine generation rate, which varies significantly between individuals. As a result, normalizing biomarker concentrations to creatinine will potentially introduce bias. There are 4 settings in particular where normalizing to creatinine could generate misleading results: 1) where there are significantly higher or lower creatinine generation rates (i.e. increased or reduced muscle mass) or more extrarenal degradation of creatinine (which is substantially higher in patients with advanced CKD); 2) in the setting of an acute change in GFR where the excretion of creatinine has not reached a steady state; 3 ) in the setting of markedly increased or decreased tubular secretion of creatinine; and 4) in low urine flow states where there may be reabsorption of creatinine in the proximal tubules.

As a result, alternative methods of urine normalization have been proposed. There is some interest in the use of alternative urinary peptides although the development of these techniques is in the very early stages. The use of short, timed urine collections has also been proposed, although this is not always practical in the ambulatory setting. Similarly, normalizing to the actual urine flow rate at the time of collection would lead to more unbiased results, although measuring this accurately is not without difficulty. Interestingly, one recent study suggested that absolute biomarker values were better at diagnosing AKI, while normalizing to creatinine was a better predictor of overall adverse outcomes. This finding may reflect the prognostic significance of creatinine generation rate, which influences the urinary creatinine concentration and therefore creatinine-normalized biomarker levels. For the moment, the majority of studies continue to normalize to creatinine, understanding that this is an imperfect technique. In a clinical setting, it should be recognized that results should be interpreted with reference to the factors that may influence the urinary creatinine concentration.

\section{Additional Readings \\ References}

32. Conti M, Moutereau S, Esmilaire L, et al. Should kidney tubular markers be adjusted for urine creatinine? the example of urinary cystatin C. Clin Chem Lab Med. 2009; 47(12):1553-1556. [PubMed: 19877765] Jantos-Siwy J, Schiffer E, Brand K, et al. Quantitative urinary proteome analysis for biomarker evaluation in chronic kidney disease. J Proteome Res. 2009; 8(1):268-281. [PubMed: 19012428]

33. Ralib AM, Pickering JW, Shaw GM, et al. Test characteristics of urinary biomarkers depend on quantitation method in acute kidney injury. J Am Soc Nephrol. 2012; 23(2):322-333. [PubMed: 22095948]

34. Waikar SS, Sabbisetti VS, Bonventre JV. Normalization of urinary biomarkers to creatinine during changes in glomerular filtration rate. Kidney Int. 2010; 78(5):486-494. [PubMed: 20555318]

\section{NOVEL BIOMARKERS IN CLINICAL CONDITIONS}

\section{Novel Biomarkers of Acute Kidney Injury}

AKI is a syndrome characterized by a rapid reduction in GFR associated with the accumulation of waste products usually excreted by the kidney. It is relatively common, occurring in up to $9 \%$ of all hospitalized patients, and is particularly prevalent in patients admitted to the Intensive Care Unit. The mortality of patients requiring dialysis in the intensive care unit is $40-60 \%$, and this has remained high over the past 20 years. Current 
definitions of AKI include a change in serum creatinine over a defined period as one of the major criteria. As mentioned above, this leads to a delay in diagnosis because of the lag time between the occurrence of the injury and a rise in serum creatinine (Figure 2). As a result, much effort is being focused on biomarkers of AKI that can make the diagnosis earlier, more accurately, and possibly enable the use of strategies to prevent progression of this syndrome.

One of the best-studied new biomarkers of AKI is NGAL. NGAL is a 21-kDa protein. It is involved in innate immunity and is expressed primarily by immune cells but also by hepatocytes and kidney tubular cells. It was originally identified through transcriptome analysis in a mouse model of ischemia-reperfusion injury where its production in the kidney tubules was noted to increase rapidly following kidney injury. It is readily detectable in the urine and resistant to degradation by proteases making it a potentially ideal biomarker of AKI. NGAL appears in the urine early after injury, and studies of cultured human tubular cells confirmed that it is produced in response to a hypoxic injury. Urinary and plasma NGAL levels have been shown to correlate with the degree of kidney injury, and levels return to baseline on resolution of AKI. Interestingly, the source of plasma and urinary NGAL appears to be different. The majority of urinary NGAL is produced in the tubules in response to injury, while most plasma NGAL originates in distant organs where its production is upregulated in the setting of AKI. The increase in plasma NGAL in this setting is at least in part related to decreased clearance due to the reduction in GFR. Commercial assays are available for the measurement of NGAL.

Urinary NGAL has been shown to be a possible early biomarker of AKI in adults and children following cardiac surgery, and after administration of radiocontrast. Similarly, elevations of NGAL predict AKI in patients presenting to the emergency room. A large meta-analysis of studies of NGAL in AKI found that there was no advantage in using urinary NGAL as opposed to serum NGAL. NGAL performed better in children, although it remained useful in adults suggesting that there may be other factors that influence NGAL levels in this population. A wide range of cut-offs for NGAL were used in the various studies, but a level of $>150 \mathrm{ng} / \mathrm{ml}$ appeared to be the most appropriate, particularly when commercial assays were used. Interestingly, normalization of the results to creatinine did not appear to affect the accuracy of the test. Elevated NGAL in the absence of elevated creatinine may be prognostically significant, and suggests that creatinine may misclassify individuals with currently subclinical kidney disease. This was borne out by a meta-analysis which found that elevations in NGAL even in the absence of an elevated creatinine predicted worse outcomes. This finding suggests that there may need to be further changes in the way that we define AKI to incorporate these biomarkers.

IL-18 is an $18 \mathrm{kDa}$ pro-inflammatory cytokine that is expressed primarily by macrophages but also by monocytes, dendritic cells and kidney epithelial cells. It plays a role in the innate and adaptive immune response and is upregulated in inflammatory states. IL-18 is thought to be one of the mediators of injury in ischemic AKI. Inhibition of IL-18 has been shown to be effective in treating inflammatory disorders in mice and interstitial IL-18 expression is increased in mouse models of AKI. Mice deficient in caspase, an IL-18-activating enzyme, develop less acute tubular necrosis than wild-type mice in models of ischemia. Urinary IL-18 originates in the kidney tubular epithelium, and thus was proposed as a potential marker of AKI.

IL-18 has been shown to rise early in patients with sepsis in the intensive care unit and was a good predictor of AKI, particularly when combined with NGAL. IL-18 increased in patients with acute tubular necrosis, but not those with pre-renal AKI, CKD, and normal controls. Similarly, rising levels of IL-18 predicted AKI in pediatric patients undergoing cardiac 
surgery. Higher post-operative urinary IL-18 has also been shown to identify AKI and predict progression of AKI following cardiac surgery in adults. Potential limitations of IL-18 derive from the fact that it may be a more generalized marker of inflammation rather than a specific marker of AKI, particularly in older age groups where there may be underlying baseline reduced kidney function.

Another biomarker that has attracted considerable interest is KIM-1. KIM-1 was initially found to be highly upregulated in a rat model of ischemic kidney injury. It is a transmembrane protein that is expressed at very low levels in the normal kidney, but its production increases in dedifferentiated proximal tubular cells in the presence of ischemic or nephrotoxic AKI. The extracellular domain of KIM-1 appears in the urine shortly following ischemic injury and can be readily detected by urinary dipstick, potentially making it a convenient and readily available marker of AKI.

Early studies in humans found that, in common with the rat, KIM-1 expression was increased in kidney biopsies from patients with acute tubular necrosis. Elevated urinary KIM-1 levels predicted an increased risk of mortality or need for dialysis in hospitalized patients with AKI and was a sensitive predictor of AKI in children undergoing cardiac surgery. Its performance as a biomarker of AKI may prove to be better in patients with normal baseline kidney function, as is the case for many novel biomarkers. This may be due to the fact that although expression is very limited in normal kidney tissue, there is upregulation of expression in various chronic kidney diseases with the degree of expression correlating with tubulointerstitial fibrosis. Its role in CKD is currently under investigation.

The kidneys contain large amounts of enzymes that perform specialized functions in tubular cells. These enzymes can be released into the urine in the event of kidney injury both as a result of leakage from cells (preformed enzymes) or by upregulation of their production in response to injury. Because they are localized to specific cells along the nephron, the presence of certain enzymes in the urine can give a clue as to the specific location of the kidney injury. $\mathrm{N}$-acetyl-glucosaminidase (NAG) is a lysosomal enzyme primarily localized to the proximal tubule. Glutathione-S-transferase (GST) is a family of enzymes with 8 different classes found throughout the nephron. However, the alpha isoform is present in proximal tubular cells alone while the pi isoform is found only in distal tubular cells. Alkaline phosphatase, GGT, alanine aminopeptidase (AAP), and lactate dehydrogenase (LDH) are brush border enzymes originating in the proximal tubule that are normally present in the urine in small quantities and increase significantly in the setting of AKI. Tubular enzymuria has been noted in patients with a broad range of kidney diagnoses including acute tubular necrosis, interstitial nephritis, nephrotoxicity and acute allograft rejection. No individual enzyme has been shown to be a consistent predictor of AKI or need for dialysis across all studies and while some have shown promise in certain homogenous populations, these results have not been replicated across heterogenous groups. This may in part be due to the fact that enzymuria is increased in many settings and is not specific for AKI. There may also be settings where the activity of the enzymes is reduced by exogenous factors, for example, while NAG has been shown to be a sensitive marker of AKI in certain situations, the presence of heavy metals and other nephrotoxins in the urine inhibits its activity reducing the sensitivity of the assay. There is a suggestion that panels of enzymes may be more useful than individual enzymes in diagnosing kidney disorders, although their primary use may lie in the diagnosis of drug nephrotoxicity where levels of these enzymes increase prior to any increase in creatinine and may help guide drug dosing.

Apart from cystatin there are a number of other proteins that are normally filtered at the glomerulus but do not appear in the urine because of uptake and metabolism in the proximal tubule. The presence of these biomarkers in the urine suggests tubular dysfunction 
(although, as mentioned previously, in the presence of sufficient albuminuria the normal uptake mechanism can be overwhelmed and they can become present even with normal tubular function). The most important of these are $\beta 2$-microglobulin and Retinol Binding Protein-4 (RBP-4). $\beta 2$-microglobulin is a $11.8 \mathrm{kDa}$ protein component of the major histocompatibility complex class I molecule that is freely filtered in the glomerulus and is reabsorbed and metabolized by the tubules with $<1 \%$ appearing in the urine. Urinary $\beta 2-$ microglobulin increases in the setting of non-selective proteinuria and also as a result of tubular damage, particularly as a result of exposure to tubular toxins. Urinary $\beta 2-$ microglobulin has been studied in a wide range of clinical contexts and appears to be useful in distinguishing pre-renal azotemia from acute tubular necrosis but is of uncertain utility in patients with sepsis where it can rise in the absence of AKI. RBP4 is primarily synthesized by hepatocytes and is involved in the transport of retinol in the blood. The C-terminally processed form is excreted in the urine and accumulates in patients with a reduced GFR. $\mathrm{RBP} 4$ is reabsorbed in the proximal tubule and appears in large quantities when there is significant proximal tubular damage. It is a particularly sensitive marker of nephrotoxicity and is elevated in reduced kidney function caused by heavy metals and is elevated in patients with diabetic nephropathy. One advantage that it carries over $\beta 2$ microglobulin is that it is more stable at low $\mathrm{pH}$.

There are a large number of other biomarkers that are under investigation to determine their utility in the early diagnosis of kidney diseases including AKI. The majority are proteins expressed in the tubules whose urinary concentrations change in the event of tubular injury. Trefoil factor 3 is a protein involved in the maintenance of the integrity of mucosal surfaces. It is normally found in the urine and decreasing levels are a sensitive marker of proximal tubular/collecting duct dysfunction, particularly due to nephrotoxicity. L-FABP is a cytoplasmic protein expressed in proximal tubules that binds free fatty acids and transports them to the mitochondria for metabolism. Increased urinary L-FABP has been noted in patients with AKI (particularly ischemic), nephrotoxicity and severe sepsis in the absence of AKI.

Osteopontin is a $44 \mathrm{kDa}$ protein primarily synthesized in bone, but also in macrophages, activated T cells, and endothelial cells. In the kidney, it is expressed in the loop of Henle and the distal tubules, and is involved in kidney protection against oxidative stress and ischemia. Increased urinary osteopontin has been noted in some chronic kidney diseases including $\operatorname{Ig} \mathrm{A}$ nephropathy, membranous nephropathy, and glomerulonephritis, as well as in patients with AKI. Netrin-1 is an axonal-guidance molecule that is not expressed in normal tubular cells but is highly expressed following kidney injury, rising within 2 hours of an acute insult. Levels correlated with the degree of injury and return to normal as the injury resolves. Clusterin is an $80 \mathrm{kDa}$ protein that is expressed constitutively during normal kidney development. Its production is upregulated in response to kidney injury and it is expressed throughout the nephron. It has shown promise as a biomarker of tubular injury and regeneration in animal models, but has not yet been investigated in large human studies.

\section{Additional Readings}

\section{References}

35. Amin AP, Salisbury AC, McCullough PA, et al. Trends in the incidence of acute kidney injury in patients hospitalized with acute myocardial infarction. Arch Intern Med. 2012; 172(3):246-253. [PubMed: 22332157]

36. Bagshaw SM, Langenberg C, Haase M, Wan L, May CN, Bellomo R. Urinary biomarkers in septic acute kidney injury. Intensive Care Med. 2007; 33(7):1285-1296. [PubMed: 17487471]

37. Bellomo R, Kellum JA, Ronco C. Acute kidney injury. Lancet. 2012 
38. Bonventre JV. Kidney injury molecule-1 (KIM-1): A urinary biomarker and much more. Nephrol Dial Transplant. 2009; 24(11):3265-3268. [PubMed: 19318357]

39. Edelstein, CL. Biomarkers in kidney disease. 1. Amsterdam ; Boston: Academic Press/Elsevier; 2011.

40. Koyner JL, Garg AX, Coca SG, et al. Biomarkers predict progression of acute kidney injury after cardiac surgery. J Am Soc Nephrol. 2012; 23(5):905-914. [PubMed: 22383693]

41. Haase M, Bellomo R, Devarajan P, Schlattmann P, Haase-Fielitz A. NGAL Meta-analysis Investigator Group: Accuracy of neutrophil gelatinase-associated lipocalin (NGAL) in diagnosis and prognosis in acute kidney injury: A systematic review and meta-analysis. Am J Kidney Dis. 2009; 54(6):1012-1024. [PubMed: 19850388]

42. Haase M, Bellomo R, Story D, Davenport P, Haase-Fielitz A. Urinary interleukin-18 does not predict acute kidney injury after adult cardiac surgery: A prospective observational cohort study. Crit Care. 2008; 12(4):R96. [PubMed: 18673539]

43. Haase M, Devarajan P, Haase-Fielitz A, et al. The outcome of neutrophil gelatinase-associated lipocalin-positive subclinical acute kidney injury: A multicenter pooled analysis of prospective studies. J Am Coll Cardiol. 2011; 57(17):1752-1761. [PubMed: 21511111]

44. Han WK, Bailly V, Abichandani R, Thadhani R, Bonventre JV. Kidney injury molecule-1 (KIM-1): A novel biomarker for human renal proximal tubule injury. Kidney Int. 2002; 62(1): 237-244. [PubMed: 12081583]

45. Liangos O, Perianayagam MC, Vaidya VS, et al. Urinary N-acetyl-beta-(D)-glucosaminidase activity and kidney injury molecule-1 level are associated with adverse outcomes in acute renal failure. J Am Soc Nephrol. 2007; 18(3):904-912. [PubMed: 17267747]

46. Lisowska-Myjak B. Serum and urinary biomarkers of acute kidney injury. Blood Purif. 2010; 29(4):357-365. [PubMed: 20389065]

47. Mishra J, Ma Q, Prada A, et al. Identification of neutrophil gelatinase-associated lipocalin as a novel early urinary biomarker for ischemic renal injury. J Am Soc Nephrol. 2003; 14(10):25342543. [PubMed: 14514731]

48. Parikh CR, Coca SG, Thiessen-Philbrook H, et al. Postoperative biomarkers predict acute kidney injury and poor outcomes after adult cardiac surgery. J Am Soc Nephrol. 2011; 22(9):1748-1757. [PubMed: 21836143]

49. Parikh CR, Jani A, Melnikov VY, Faubel S, Edelstein CL. Urinary interleukin-18 is a marker of human acute tubular necrosis. Am J Kidney Dis. 2004; 43(3):405-414. [PubMed: 14981598]

50. Parikh CR, Mishra J, Thiessen-Philbrook H, et al. Urinary IL-18 is an early predictive biomarker of acute kidney injury after cardiac surgery. Kidney Int. 2006; 70(1):199-203. [PubMed: 16710348] Urbschat A, Obermuller N, Haferkamp A. Biomarkers of kidney injury. Biomarkers. 2011; 16(Suppl 1):S22-30. [PubMed: 21707441]

51. Waikar SS, Betensky RA, Bonventre JV. Creatinine as the gold standard for kidney injury biomarker studies? Nephrol Dial Transplant. 2009; 24(11):3263-3265. [PubMed: 19736243]

52. Waring WS, Moonie A. Earlier recognition of nephrotoxicity using novel biomarkers of acute kidney injury. Clin Toxicol (Phila). 2011; 49(8):720-728. [PubMed: 21970770]

53. $\mathrm{Yu}$ Y, Jin $\mathrm{H}$, Holder D, et al. Urinary biomarkers trefoil factor 3 and albumin enable early detection of kidney tubular injury. Nat Biotechnol. 2010; 28(5):470-477. [PubMed: 20458317]

\section{Novel Biomarkers of CKD}

CKD affects approximately $11 \%$ of the US population and is a major contributor to morbidity and mortality. Early identification of those at risk of CKD is important and has been facilitated by the routine use of estimating equations for GFR (MDRD Study equation / CKD-EPI equation). The classification of CKD into stages and stratification by degree of proteinuria has helped identify patients at risk of progression to end-stage renal disease. However, current techniques for estimating GFR rely on serum creatinine with all its attendant deficiencies and newer biomarkers are being sought that may better stratify patients by risk category. In common with AKI, no single biomarker has been identified that can reliably predict progression of CKD. 
As discussed above, creatinine and cystatin $\mathrm{C}$ are the biomarkers used most often to determine GFR. Another promising marker of GFR is $\beta$-trace protein (BTP). BTP is a low molecular mass protein belonging to the lipocalin protein family that is produced at a constant rate by glial cells in the central nervous system. It is freely filtered at the glomerulus and is reabsorbed and metabolized in the proximal tubule. There is almost no non-renal elimination. It has been shown to be as good as cystatin $\mathrm{C}$ and creatinine at detecting mild abnormalities in kidney function and is also better than creatinine at predicting adverse outcomes in patients with acute heart failure. Estimating equations are being derived that include serum BTP as the predictor, although they have not been validated in large studies. BTP can aid in the prediction of progression of CKD but, similar to cystatin C, corticosteroids can influence levels independent of GFR.

As well as its potential role in the diagnosis of AKI, NGAL may also be a useful biomarker in patients with CKD, particularly for identifying patients at risk of a significant decline in GFR, because of its ability to detect subtle changes in tubular function. Urinary and serum NGAL levels are elevated in a wide range of kidney diseases including IgA nephropathy, autosomal dominant polycystic kidney disease and diabetic nephropathy. Urinary NGAL has been shown to differentiate HIV nephropathy from other forms of kidney disease while higher levels were associated with an increased risk of progression in a diverse group of patients with CKD.

$\mathrm{AKI}$ is itself a risk factor for future $\mathrm{CKD}$, but there is no reliable means of determining who will recover entirely and who will be left with some kidney impairment following an episode of AKI. NGAL is a potential marker of future progression. Similarly, KIM-1 may have a role in predicting the transition from AKI to CKD. Urinary KIM-1 levels have been shown to correlate with proteinuria, decreasing in response to treatment with angiotensin converting enzyme (ACE) inhibitors or a low sodium diet, suggesting a potential role as a target for determining therapeutic efficacy.

Urinary L-FABP correlates with the degree of proteinuria in patients with CKD. In patients with diabetes, it has been shown to correlate with GFR and predict progression to end-stage renal disease. Baseline levels predicted the future development of albuminuria, suggesting a potential role in stratifying patients who might benefit from early preventative therapies. Other potential urinary biomarkers of CKD include connective tissue growth factor, L-type fatty acid binding protein, and trefoil factor 3 .

Asymmetric dimethylarginine (ADMA) is an inhibitor of nitric oxide synthase and a marker of endothelial function. Increasing plasma levels predict progression of CKD and mortality in patients with chronic kidney failure in diabetic and non-diabetic kidney disease. Increased plasma levels are also predictive of future cardiovascular mortality in patients with known CKD and cardiovascular disease.

Fibroblast growth factor-23 (FGF-23) is a phosphaturic hormone that is elevated in patients with CKD. The degree of elevation correlates with the stage of CKD, and elevations in FGF-23 are noted prior to any elevation in phosphate.

In order to be useful for clinical practice, new biomarkers of CKD progression will have to prove superior to the impressive prognostic ability of eGFR and albuminuria. New biomarkers of GFR estimation will have to improve precision, bias, and prognostic ability of creatinine and cystatin $\mathrm{C}$.

Studies from the NIH Chronic Kidney Disease Biomarkers Consortium and other investigators are underway to identify and validate novel biomarkers of CKD. 


\section{Additional Readings}

References

54. Bolignano D, Lacquaniti A, Coppolino G, et al. Neutrophil gelatinase-associated lipocalin (NGAL) and progression of chronic kidney disease. Clin J Am Soc Nephrol. 2009; 4(2):337-344. [PubMed: 19176795]

55. Fassett RG, Venuthurupalli SK, Gobe GC, Coombes JS, Cooper MA, Hoy WE. Biomarkers in chronic kidney disease: A review. Kidney Int. 2011; 80(8):806-821. [PubMed: 21697815]

56. Kamijo-Ikemori A, Sugaya T, Yasuda T, et al. Clinical significance of urinary liver-type fatty acidbinding protein in diabetic nephropathy of type 2 diabetic patients. Diabetes Care. 2011; 34(3): 691-696. [PubMed: 21273494]

57. Lu TM, Chung MY, Lin CC, Hsu CP, Lin SJ. Asymmetric dimethylarginine and clinical outcomes in chronic kidney disease. Clin J Am Soc Nephrol. 2011; 6(7):1566-1572. [PubMed: 21642363]

58. Manzano-Fernandez S, Januzzi JL, Boronat-Garcia M, et al. Impact of kidney dysfunction on plasma and urinary $\mathrm{N}$-terminal pro-B-type natriuretic peptide in patients with acute heart failure. Congest Heart Fail. 2010; 16(5):214-220. [PubMed: 20887618]

59. Priem F, Althaus H, Jung K, Sinha P. Beta-trace protein is not better than cystatin C as an indicator of reduced glomerular filtration rate. Clin Chem. 2001; 47(12):2181. [PubMed: 11719491]

60. Ravani P, Tripepi G, Malberti F, Testa S, Mallamaci F, Zoccali C. Asymmetrical dimethylarginine predicts progression to dialysis and death in patients with chronic kidney disease: A competing risks modeling approach. J Am Soc Nephrol. 2005; 16(8):2449-2455. [PubMed: 15944335]

61. Spanaus KS, Kollerits B, Ritz E, et al. Serum creatinine, cystatin C, and beta-trace protein in diagnostic staging and predicting progression of primary nondiabetic chronic kidney disease. Clin Chem. 2010; 56(5):740-749. [PubMed: 20224047]

62. Waanders F, Vaidya VS, van Goor H, et al. Effect of renin-angiotensin-aldosterone system inhibition, dietary sodium restriction, and/or diuretics on urinary kidney injury molecule 1 excretion in nondiabetic proteinuric kidney disease: A post hoc analysis of a randomized controlled trial. Am J Kidney Dis. 2009; 53(1):16-25. [PubMed: 18823687]

\section{Biomarkers of Nephrotoxicity}

Nephrotoxicity is an unfortunate side effect of many drugs, and is a relatively common cause of acute and chronic kidney injury. Currently, clinical nephrotoxicity is diagnosed when there is an increase in the serum creatinine concentration or a reduction in the urine output. These are relatively late complications of drug-induced kidney injury, and are also non-specific. Often, nephrotoxicity is a diagnosis of exclusion when other causes are ruled out. Nephrotoxicity can be localized to a specific part of the nephron which the serum creatinine can not distinguish. Proteinuria (e.g. due to vascular endothelial growth factor inhibitors) and subtle signs of defects in proximal tubular function (e.g. related to the use of antiretroviral medications) can suggest specific forms of nephrotoxicity, but these generally arise late in the process. Specific biomarkers that rise earlier in the course of nephrotoxicity and more accurately localize the site of the lesion would be of great benefit both in the clinical management of nephrotoxicity (by identifying patients at risk and allowing the withdrawal of an offending agent) as well as in drug development. As it is a defined insult at a specific time, drug nephrotoxicity is a good model for the study of AKI and there is much ongoing research in this field.

NAG is produced by proximal tubular cells in response to ischemic and oxidative stress. In animal studies, increased urinary NAG is a sensitive marker of gentamicin and cisplatin toxicity with levels decreasing following antioxidant therapy suggesting a potential role for this class of drugs in some cases. Other tubular enzymes are also potential markers of nephrotoxicity. Increased GST-alpha levels suggest proximal tubular damage in models of methotrexate-induced kidney damage, and GST-pi is a marker of distal tubular dysfunction. GGT, AAP, and LDH all increase in the setting of gentamicin and vancomycin use, in the 
absence of a reduction in GFR suggesting a potential role in monitoring for potential toxicity. Urinary calbindin-D may be a marker of distal renal tubular injury caused by cisplatin-based chemotherapy.

One study evaluated the performance of a panel of biomarkers in a model of acute and subacute gentamicin toxicity. The panel included urinary cystatin C, NGAL, KIM-1, $\beta 2$ microglobulin, clusterin, GST-alpha, and osteopontin. Urinary cystatin C and NGAL were the most sensitive markers of gentamicin toxicity with changes appearing within one day, while KIM-1 levels best correlated with histological appearance in the subacute model. In another model of gentamicin nephrotoxicity, urinary KIM-1 levels rose within 24 hours of administration, while urinary NAG and serum blood urea nitrogen and creatinine remained in the normal range. This was despite histologic evidence of necrosis in half of the kidney tubules, thus elegantly illustrating the need for more sensitive biomarkers that the traditional panel.

As noted earlier, FDA has qualified the following biomarkers-urinary KIM-1, albumin, total protein, B2-microglobulin, cystatin C, clusterin, trefoil factor-3, and renal papillary antigen-1-along with traditional clinical chemistry markers and histopathology for the detection of acute drug-induced nephrotoxicity in rat toxicology studies.

\section{Additional Readings}

\section{References}

63. Ali BH, Al-Salam S, Al-Husseini I, Nemmar A. Comparative protective effect of N-acetyl cysteine and tetramethylpyrazine in rats with gentamicin nephrotoxicity. J Appl Toxicol. 2009; 29(4):302307. [PubMed: 19117019]

64. Hoffmann D, Fuchs TC, Henzler T, et al. Evaluation of a urinary kidney biomarker panel in rat models of acute and subchronic nephrotoxicity. Toxicology. 2010; 277(1-3):49-58. [PubMed: 20816719]

65. Waring WS, Moonie A. Earlier recognition of nephrotoxicity using novel biomarkers of acute kidney injury. Clin Toxicol (Phila). 2011; 49(8):720-728. [PubMed: 21970770]

66. Zhou Y, Vaidya VS, Brown RP, et al. Comparison of kidney injury molecule-1 and other nephrotoxicity biomarkers in urine and kidney following acute exposure to gentamicin, mercury, and chromium. Toxicol Sci. 2008; 101(1):159-170. [PubMed: 17934191]

\section{Biomarkers of Glomerular Disease}

The clinical course of glomerular diseases is variable and is difficult to predict without serial kidney biopsies. Treatments are generally toxic, and repeated courses of immunosuppression are sometimes indicated in relapsing cases. An ideal biomarker in patients with glomerular diseases would be one that gives information about disease activity, prognosis, and likelihood of clinical relapse.

This is particularly relevant in patients with lupus nephritis. Lupus nephritis is a highly variable disease with multiple classes requiring different treatments. Patients can move from one class to another, and rates of relapse are high. Biomarkers that could non-invasively identify the class of lupus, predict response to therapy, and alert to relapse could revolutionize treatment. Urinary NGAL has been investigated as a potential biomarker of disease activity in patients with lupus. It has been shown to predict kidney flares better than traditional methods without any correlation with extra-renal disease. Urinary NGAL was also higher in patients with lupus nephritis than those with clinical lupus without kidney involvement. A recent study examined a panel of biomarkers in a diverse group of patients with lupus nephritis and found that the combination of urinary NGAL and monocyte chemotactic peptide-1 (MCP-1) was an excellent test of lupus nephritis chronicity, while the 
combination of MCP-1, a-1-acid glycoprotein, and ceruloplasmin predicted disease activity. MCP-1 is a chemotactic chemokine that is specific for monocytes. Increased urinary levels have been noted in patients at the time of lupus flares, and the increase can be seen up to 4 months before the clinical flare suggesting that is a potential predictor. A recent pilot study has suggested that a combination of urinary proteins and clinical variables could be used to derive a potentially useful composite biomarker panel that reflects specific pathologic lesions such as tubulointerstitial inflammation in lupus nephritis. Currently, no single biomarker can definitively predict a lupus flare. Because the treatment is relatively toxic, the specificity of any predictive test would have to be very high to justify its use. It is possible that a suitable panel of biomarkers may be developed that will allow for this degree of certainty.

Apart from predicting relapses, another potential benefit of biomarkers would be to noninvasively determine which class of lupus is present in patients with known lupus nephritis. Currently, this is done by examining the urine sediment and measuring proteinuria. The presence of red cell casts suggests a class III or IV nephritis while heavy proteinuria is more suggestive of membranous lupus. This is not an exact science and it is not unusual for a kidney biopsy to be unexpectedly abnormal in a patient with a relatively benign urine sediment. A number of biomarkers are being investigated for their potential in identifying lupus class. A recent paper examined the urine metabolomic profiles of patients with different classes of lupus and found that patients with class III/IV lupus had significantly lower taurine levels than controls and patients with other classes of lupus. Patients with class V lupus had significantly lower urinary citrate levels. Urinary CXCR3, interferon-producing protein-10, transforming growth factor- $\beta$, and vascular endothelial growth factor was also able to differentiate class IV lupus from other classes with urinary IP-10 performing the best. None of these tests are reliable enough currently to take the place of a kidney biopsy at present.

IgA nephropathy is the most common glomerulonephritis, and is a frequent cause of endstage renal disease. The clinical course of $\operatorname{IgA}$ nephropathy is very variable; the presence of crescents on kidney biopsy suggests a more aggressive disease with a poorer prognosis and mandates the use of aggressive immunosuppression. Currently, patients are stratified according to the presence of specific histologic features on kidney biopsy and the degree of proteinuria at presentation. Many biomarkers are being investigated to determine their utility in predicting which patients will progress to chronic kidney failure and earlier stages of $\mathrm{CKD}$, and the overall response to therapy without resorting to follow-up biopsies. One study of patients with crescentic IgA found that combining the number of preserved glomeruli on biopsy with the serum creatinine at baseline and the fractional excretion of $\mathrm{IgG}$ was able to classify patients as responders or non-responders to therapy in all cases. One limitation of this study was of course the requirement for a kidney biopsy. More recently, urinary IL-18 has been shown to discriminate patients who progress from those who remain stable. NGAL and NAG are elevated in patients with IgA nephropathy and significant tubulointerstitial disease. IL-6 is a cytokine expressed by antigen-presenting cells that has been shown to be elevated in the urine of patients with active lupus nephritis. Similarly, elevated urinary IL-6 has been noted in patients with progressive $\operatorname{IgA}$ nephropathy relative to controls, suggesting ongoing intra-renal inflammation. Cytokines including MCP-1 have also been shown to be increased in patients with progressive disease.

Anti-neutrophil cytoplasmic antibody (ANCA) disease is a pauci-immune crescentic vasculitis that commonly affects the kidneys and is associated with a high risk of relapse. An active urinary sediment and an increasing ANCA titer are traditional biomarkers of relapse but these can be unreliable. Recently, a proteome analysis of the urine of patients with ANCA disease and matched controls found that a panel of 47 biomarkers could reliably 
distinguish ANCA disease from other causes of kidney dysfunction and also correlated with disease activity and response to therapy. The majority of these biomarkers were related to breakdown products of hemoglobin or albumin.

The diagnosis and treatment of membranous nephropathy may be aided in the future by the discovery that approximately $80 \%$ of patients with primary membranous nephropathy have IgG4 antibodies to the M-type phospholipase A2 receptor in their serum that is not present in normal controls. Levels of this antibody correlate with disease activity and response to treatment. Interestingly, the presence of these antibodies in patients prior to transplant does not necessarily predict recurrence, suggesting that there is an interplay of risk factors that predisposes to this disease. Other autoantibodies including antibodies to bovine serum albumin and superoxide dismutase are being evaluated for their roles in the pathogenesis of idiopathic membranous nephropathy. Other biomarkers that may play a role in understanding the pathogenesis, clinical identification, and management of patients with nephrotic syndrome include circulating soluble urokinase receptor in focal segmental glomerulosclerosis and podocyte-secreted angiopoietin-like-4 in minimal change disease.

\section{Additional Readings}

\section{References}

67. Avihingsanon Y, Phumesin P, Benjachat T, et al. Measurement of urinary chemokine and growth factor messenger RNAs: A noninvasive monitoring in lupus nephritis. Kidney Int. 2006; 69(4): 747-753. [PubMed: 16518330]

68. Bazzi C, Rizza V, Raimondi S, Casellato D, Napodano P, D’Amico G. In crescentic IgA nephropathy, fractional excretion of IgG in combination with nephron loss is the best predictor of progression and responsiveness to immunosuppression. Clin J Am Soc Nephrol. 2009; 4(5):929935. [PubMed: 19406958]

69. Brunner HI, Bennett MR, Mina R, et al. Non-invasive renal protein biomarkers are associated with histological features of lupus nephritis. Arthritis Rheum. 2012; 64(8):2687-2697. [PubMed: 22328173]

70. Haubitz M, Good DM, Woywodt A, et al. Identification and validation of urinary biomarkers for differential diagnosis and evaluation of therapeutic intervention in anti-neutrophil cytoplasmic antibody-associated vasculitis. Mol Cell Proteomics. 2009; 8(10):2296-2307. [PubMed: 19564150]

71. Herrmann SM, Sethi S, Fervenza FC. Membranous nephropathy: The start of a paradigm shift. Curr Opin Nephrol Hypertens. 2012; 21(2):203-210. [PubMed: 22240444]

72. Pitashny M, Schwartz N, Qing X, et al. Urinary lipocalin-2 is associated with renal disease activity in human lupus nephritis. Arthritis Rheum. 2007; 56(6):1894-1903. [PubMed: 17530720]

73. Romick-Rosendale LE, Brunner HI, Bennett MR, et al. Identification of urinary metabolites that distinguish membranous lupus nephritis from proliferative lupus nephritis and focal segmental glomerulosclerosis. Arthritis Res Ther. 2011; 13(6):R199. [PubMed: 22152586]

74. Rubinstein T, Pitashny M, Levine B, et al. Urinary neutrophil gelatinase-associated lipocalin as a novel biomarker for disease activity in lupus nephritis. Rheumatology (Oxford). 2010; 49(5):960 971. [PubMed: 20144927]

75. Stangou M, Alexopoulos E, Papagianni A, et al. Urinary levels of epidermal growth factor, interleukin-6 and monocyte chemoattractant protein-1 may act as predictor markers of renal function outcome in immunoglobulin A nephropathy. Nephrology (Carlton). 2009; 14(6):613-620. [PubMed: 19143943]

76. Wei C, El Hindi S, Li J, et al. Circulating urokinase receptor as a cause of focal segmental glomerulosclerosis. Nat Med. 2011; 17(8):952-960. [PubMed: 21804539]

77. Zhang X, Nagaraja HN, Nadasdy T, et al. A composite urine biomarker reflects interstitial inflammation in lupus nephritis biopsies. Kidney Int. 2012; 81(4):401-406. [PubMed: 21993584] 


\section{Future Challenges}

The convergence of basic science investigations and modern clinical epidemiologic techniques has ushered in an era of discovery and early validation of a number of novel biomarkers of kidney disease. A number of challenges face clinicians and scientists before widespread adoption of novel biomarkers of kidney disease in the clinic. These include the need for larger studies with clinically important endpoints, comparisons of conventional versus novel biomarkers for their added clinical utility, correlation of severity of disease with biomarker levels and duration of elevation, clarification of the treatment implications from measurement of novel biomarkers, and development of reliable assays and point-ofcare testing if appropriate.

\section{Acknowledgments}

Support: Dr Waikar is supported by grants DK093574, DK085660, and DK075941 from the National Institute of Diabetes and Digestive Kidney Diseases. 


\section{Box 1}

\section{Characteristics of an ideal biomarker}

1. Non-invasive, easily measured, inexpensive and provides rapid results

2. From easily available sources (blood or urine)

3. High sensitivity

4. High specificity

5. Allows early detection of disease and changes in response to treatment

6. Predicts prognosis and allows stratification into categories of risk

7. Biologically plausible - provides information about the mechanisms of disease Adapted from Edelstein CL: Biomarkers in kidney disease. 1st. ed. Amsterdam; Boston, Academic Press/Elsevier, 2011 


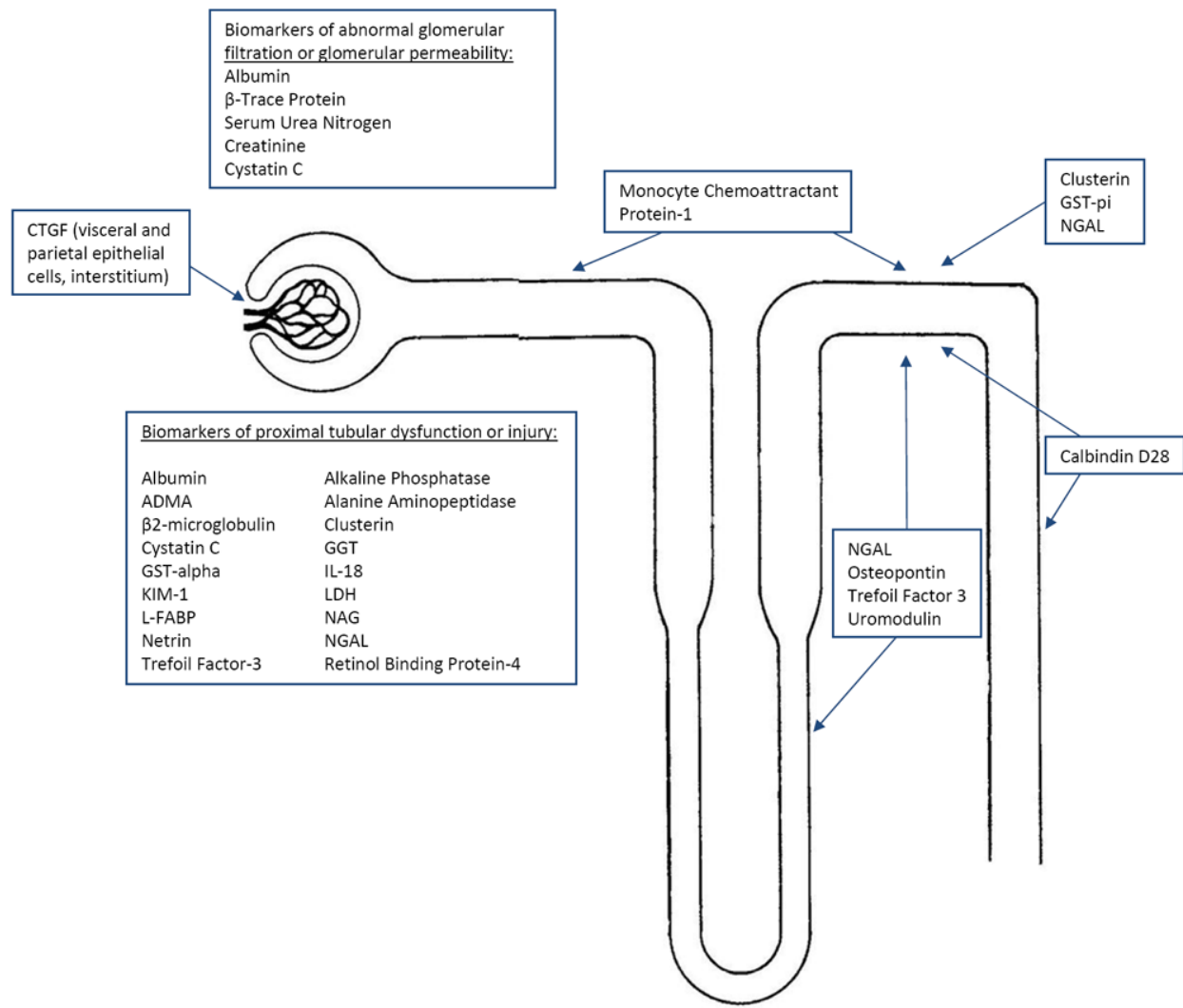

Figure 1.

Anatomical localization of biomarkers along the nephron. Abbreviations: GST, Glutathiones-transferases; CTGF, Connective tissue growth factor; NGAL, Neutrophil gelatinaseassociated lipocalin; ADMA, Asymmetric dimethyl arginine; KIM-1, Kidney injury molecule 1; L-FABP, Liver-type fatty acid binding protein; GGT, Gamma-glutamyl transpeptidase; IL-18, interleukin 18; LDH, lactate dehydrogenase; NAG, N-acetyl glucosaminidase 


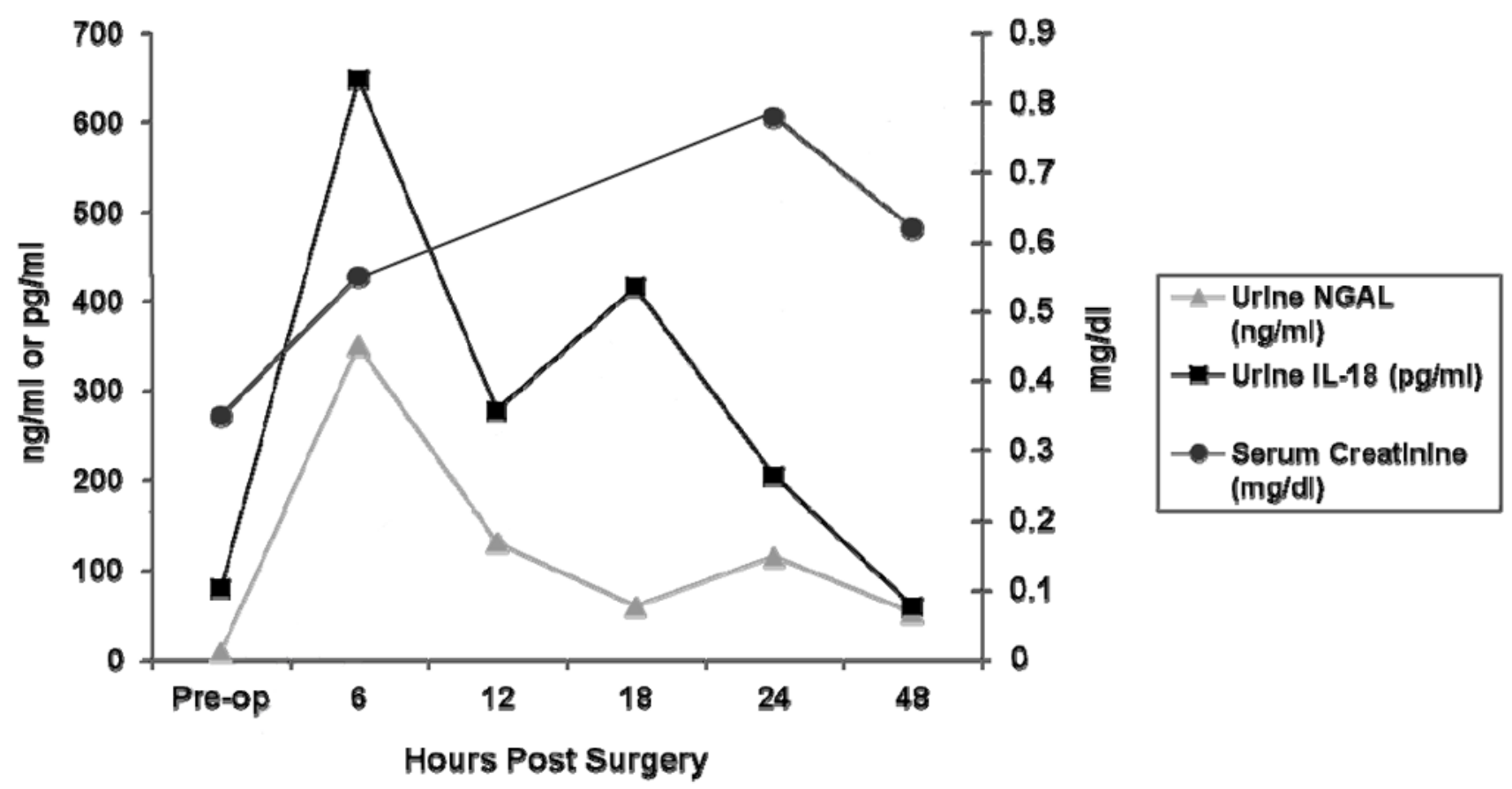

Figure 2.

Pattern of change in biomarkers over time in patients with AKI following cardiac surgery. Abbreviations: NGAL, Neutrophil gelatinase-associated lipocalin. Conversion factor for units: serum creatinine in $\mathrm{mg} / \mathrm{dL}$ to $\mu \mathrm{mol} / \mathrm{L}, \times 88.4$

Data from Parikh CR et al: Postoperative Biomarkers Predict Acute Kidney Injury and Poor Outcomes after Pediatric Cardiac Surgery. JASN. 22: 1737-1747. 
Table 1

Uses and limitations of conventional biomarkers

\begin{tabular}{|c|c|c|}
\hline & Uses & Limitations \\
\hline Creatinine & $\begin{array}{ll}\text { - } & \text { Glomerular filtration marker } \\
\text { - } & \text { eGFR estimation } \\
\text { - } & \begin{array}{l}\text { Biomarker of acute and chronic } \\
\text { reduced kidney function }\end{array}\end{array}$ & $\begin{array}{ll}\text { - } & \text { Variability in generation rates across } \\
\text { individuals } \\
\text { - } & \begin{array}{l}\text { Significant tubular secretion leading to } \\
\text { overestimation of GFR }\end{array} \\
\text { - } & \text { Significant extra-renal elimination } \\
\text { - } & \text { Tubular reabsorption in low urine flow } \\
\text { states } \\
\text { - } & \text { Increases late after AKI }\end{array}$ \\
\hline Cystatin C & $\begin{array}{ll}\text { - } & \text { GFR estimation (plasma) } \\
\text { - } & \text { Biomarker of proximal tubular } \\
\text { dysfunction (urine) }\end{array}$ & $\begin{array}{l}\text { - Increases late after AKI } \\
\text { May increase in inflammatory states and } \\
\text { where there is thyroid dysfunction } \\
\text { independent of kidney function } \\
\text { - Urinary Cystatin C is altered in the presence } \\
\text { of albuminuria }\end{array}$ \\
\hline Albuminuria & 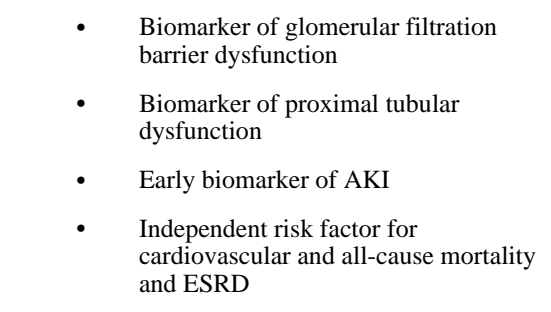 & $\begin{array}{l}\text { - } 24 \text { hour collections unreliable } \\
\text { - } \quad \text { Significant intra-individual variability in } \\
\text { albumin/creatinine ratio over short time } \\
\text { periods }\end{array}$ \\
\hline Urine sediment examination & $\begin{array}{ll}\text { - } & \text { Biomarker of AKI } \\
\text { - } & \text { Biomarker of glomerular disease } \\
\text { - } & \begin{array}{l}\text { Biomarker of tubulointerstitial } \\
\text { disease }\end{array}\end{array}$ & 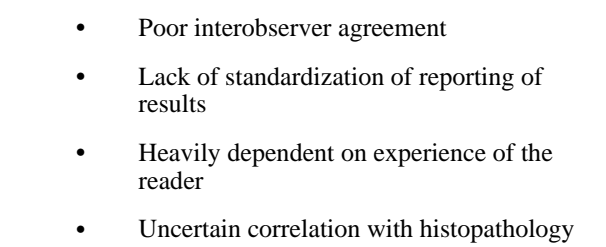 \\
\hline
\end{tabular}

Abbreviations: AKI, acute kidney injury; eGFR, estimated glomerular filtration rate ESRD, end-stage renal disease 


\section{Table 2}

Scoring system for the urine sediment based on the numbers of granular casts and kidney tubular epithelial cells.

\begin{tabular}{|c|c|c|c|}
\hline & \multicolumn{3}{|c|}{ Granular Casts per HPF } \\
\hline Kidney tubular epithelial cells per hpf & 0 (0 points) & $1-5(1$ point $)$ & $>6(2$ points $)$ \\
\hline 0 (0 points) & 0 & 1 & 2 \\
\hline $1-5$ (1 point) & 1 & 2 & 3 \\
\hline$ช 6$ (2 points) & 2 & 3 & 4 \\
\hline
\end{tabular}

Abbreviation: HPF, high-power field.

Adapted from Perazella MA, et al: Urine microscopy is associated with severity and worsening of acute kidney injury in hospitalized patients. Clin J Am Soc Nephrol 5(3):402-408, 2010 
Table 3

Examples of clinical settings in which novel biomarkers of kidney disease have been studied in humans

\begin{tabular}{|l|l|}
\hline Scenario & Selected biomarkers \\
\hline Cardiac Surgery & $\beta 2 \mathrm{M}, \mathrm{CyC}, \mathrm{GST}, \mathrm{IL}-18, \mathrm{KIM}-1, \mathrm{~L}-\mathrm{FABP}, \mathrm{NAG}, \mathrm{Netrin}, \mathrm{NGAL}$ \\
\hline Intensive Care Unit & $\beta 2 \mathrm{M}, \mathrm{CyC}, \mathrm{GST}, \mathrm{IL}-18, \mathrm{KIM}-1, \mathrm{~L}-\mathrm{FABP}, \mathrm{NAG}, \mathrm{NGAL}$ \\
\hline Contrast-induced nephropathy & $\mathrm{CyC}, \mathrm{IL}-18, \mathrm{KIM}-1, \mathrm{~L}-\mathrm{FABP}, \mathrm{NAG}, \mathrm{NGAL}$ \\
\hline Drug-induced nephrotoxicity & $\begin{array}{l}\text { AAP, AP, } \beta 2 \mathrm{M}, \mathrm{CyC}, \text { calbindin D, clusterin, GGT, GST, KIM-1, LDH, L-FABP, NAG, NGAL, osteopontin, } \\
\text { RBP4 }\end{array}$ \\
\hline Chronic Kidney Disease & ADMA, $\beta$-TP, CTGF, FGF-23, KIM-1, L-FABP, NGAL, RBP4, TFF3, uromodulin \\
\hline Glomerular Disease & IL-18, IP-10, NGAL, MCP-1, NAG, osteopontin, TGF- $\beta$ \\
\hline
\end{tabular}

Abbreviations

$\begin{array}{ll}\text { AAP } & \text { Alanine aminopeptidase } \\ \text { ADMA } & \text { Asymmetric dimethyl arginine } \\ \text { AP } & \text { Alkaline phosphatase } \\ \beta \text {-TP } & \text { Beta-trace protein } \\ \beta 2 \mathrm{M} & \text { Beta-2-microglobulin } \\ \text { CyC } & \text { Cystatin C } \\ \text { CTGF } & \text { Connective tissue growth factor } \\ \text { FGF-23 } & \text { Fibroblast growth factor 23 } \\ \text { GGT } & \text { Gamma-glutamyl transpeptidase } \\ \text { GST } & \text { Glutathione-s-transferases } \\ \text { IL-18 } & \text { Interleukin 18 } \\ \text { IP-10 } & \text { Interferon gamma-induced protein10 } \\ \text { KIM-1 } & \text { Kidney injury molecule 1 } \\ \text { L-FABP } & \text { Liver-type fatty acid binding protein } \\ \text { LDH } & \text { Lactate dehydrogenase } \\ \text { MCP-1 } & \text { Monocyte chemoattractant protein 1 } \\ \text { NAG } & \text { N-acetyl glucosaminidase } \\ \text { NGAL } & \text { Neutrophil gelatinase-associated lipocalin } \\ \text { RBP4 } & \text { Retinol binding protein } 4 \\ \text { TFF3 } & \text { Trefoil factor 3 } \\ \text { TGF- } \beta & \text { Transforming growth factor beta } \\ \end{array}$

